\title{
Secuelas tras inyección ilegal de silicona líquida como técnica de aumento mamario: presentación de 2 casos
}

\author{
María Cerveraa ${ }^{a}$ Fernando Martínez-Regueira ${ }^{a}$, Josu Sola ${ }^{b}$, Víctor Valentía, Carlos Pastora ${ }^{a}$ Ignacio Povedaa ${ }^{\text {, Pablo Martía }}$ \\ y, Gerardo Zornoza ${ }^{a}$ \\ aÁrea de Patología Mamaria. Departamento de Cirugía General y Aparato Digestivo. Clínica Universitaria. Universidad de Navarra. \\ Pamplona. Navarra. España. \\ bServicio de Anatomía Patológica. Clínica Universitaria. Universidad de Navarra. Pamplona. Navarra. España.
}

\begin{abstract}
Resumen
La utilización de silicona líquida con el fin de aumentar el volumen mamario tuvo una amplia difusión en la década de los años sesenta del siglo pasado, pero a finales de ésta, tras la publicación de numerosos estudios que describían la aparición de un gran número de complicaciones locales, así como la migración a distancia de pequeñas cantidades de silicona, su práctica se abandonó. Su empleo dificulta posteriormente el diagnóstico temprano del cáncer de mama, por lo que este grupo de pacientes no debe incluirse en los programas habituales de cribado, sino que han de seguir controles periódicos y exhaustivos. En este contexto, la resonancia magnética es la mejor prueba de imagen para la detección temprana de procesos malignos mamarios. La presencia de complicaciones locales, la sospecha de una lesión maligna o el deseo de la paciente por evitar ambas situaciones son indicación de la mastectomía subcutánea.
\end{abstract}

Palabras clave: Silicona líquida. Resonancia magnética. Cáncer de mama. Mastectomía bilateral.

\section{SEQUELAE AFTER ILLEGAL INJECTION OF LIQUID SILICONE FOR BREAST AUGMENTATION: REPORT OF TWO CASES}

The use of liquid silicone for breast augmentation was widespread in the 1960s but was abandoned at the end of the decade due to numerous studies describing the development of a large number of local complications, as well as remote migration of small amounts of silicone. The use of liquid silicone also leads to enormous difficulty in the early diagnosis of breast cancer; these patients are precluded from routine screening programs and must undergo exhaustive periodic examinations. Magnetic resonance imaging has become the most effective test for the early detection of breast cancer in these patients. Indications for subcutaneous mastectomy are the presence of local complications, suspicion of a malignant lesion, or the patient's desire to prevent both these potential problems.

Key words: Liquid silicone. Magnetic resonance imaging. Breast cancer. Bilateral mastectomy.

\section{Introducción}

Las siliconas son polímeros de dióxido de silicio cuyas características varían según la longitud de la cadena central y del radical orgánico presente en las cadenas laterales. Sus propiedades físicas varían en función del número

Correspondencia: M. Cervera Delgado.

Área de Patología Mamaria. Departamento de Cirugía Gral.

y Aparato Digestivo.

Clínica Universitaria. Universidad de Navarra.

Av. Pío XII, 36. 31008 Pamplona.

Correo electrónico: mcervdel@yahoo.es

Manuscrito recibido el 27-4-2005 y aceptado el 11-7-2005. de enlaces cruzados entre las moléculas de silicio, por lo que se obtienen siliconas fluidas en el caso de cadenas cortas, y geles o elastómeros cuando aumenta el número de unidades, y son las estructuras ramificadas y largas las que dan lugar a las formas de presentación más sólidas'.

Esta versatilidad ha permitido su uso médico, tanto en estado sólido, como en gel, o para inyección líquida. En la década de los años sesenta y setenta del siglo pasado, la inyección intramamaria de silicona líquida fue un procedimiento muy difundido como técnica de aumento mamario ${ }^{2}$, dada la sencillez y su bajo coste. La descripción temprana de múltiples complicaciones relacionadas con ella ${ }^{3,4}$ llevaron a su abandono y a la prohibición de su utilización ${ }^{5}$. En la actualidad siguen apareciendo casos 
en los que este proceder se repite en medios no médicos, habitualmente clandestinos, como en los 2 casos que presentamos.

\section{Caso clínico}

Presentamos 2 casos de pacientes de 39 y 41 años. Ambas referían la inyección de aproximadamente $250 \mathrm{ml}$ de silicona líquida, en el curso de 3-4 sesiones, como técnica de aumento mamario por personal extramédico. En el momento de la consulta referían dolor mamario y solicitaban la extirpación mamaria. En ambos casos, los exámenes practicados fueron similares. La exploración física mostró unas mamas con ligera ptosis; nódulos múltiples, fluctuantes, de hasta $3 \mathrm{~cm}$, y pequeñas adenopatías axilares, móviles.

Las mamografías mostraron imágenes de alta densidad, difusas, que imposibilitaban la visión de la glándula mamaria (fig. 1). El estudio ecográfico mamario bilateral apareció muy artefactado. El análisis con ambas técnicas, mamografía y ultrasonidos, se consideró como no valorable para la exclusión de patología mamaria neoplásica. En la resonancia magnética $(\mathrm{RM})$ se observó múltiples imágenes ovoideas, de diferente tamaño, distribuidas por el tejido graso intramamario y en el subcutáneo, llegando a contactar con la dermis (fig. 2), así como en el ámbito extramamario. Tras la administración de gadolinio no se evidenciaron captaciones focales sospechosas. A la vista de los resultados de las citadas exploraciones, se expuso a las pacientes las alternativas (controles periódicos mediante RM o cirugía). A petición de las pacientes se practicó mastectomía subcutánea bilateral, con reconstrucción inmediata mediante prótesis subpectoral. La técnica quirúrgica mostró dificultades para la correcta disección en la zona subcutánea por la importante reacción inflamatoria-necrótica existente.

\section{Estudio anatomopatológico}

Las piezas quirúrgicas, tanto en la superficie externa como al corte seriado, mostraron múltiples cavidades quísticas, con contenido oleoso translúcido, entremezclado con el tejido adiposo mamario (fig. 3), así como restos fibrosos de localización retroareolar. De la cola axilar se aislaron entre 2 y 5 ganglios linfáticos. Microscópicamente entre los lóbulos grasos aparecía un gran número de cavidades quísticas delimitadas por una única banda de células de tipo inflamatorio (fig. 4), principalmente macrófagos y abundantes células gigantes multinucleadas de cuerpo extraño, aplanadas y en algunas áreas acompañadas de una banda de fibrosis sin otras células acompañantes. En el citoplasma de los macrófagos se observaron múltiples vacuolas, que en ocasiones contenían una pequeña cantidad de materia refringente, así como múltiples áreas de necrosis grasa. El parénquima mamario no mostró alteraciones relevantes. En los ganglios axilares se observaron depósitos materiales intracelulares o extracelulares, preferentemente en el seno subcapsular, similares a las descritas en el parénquima mamario.

\section{Discusión}

En las décadas de los años cincuenta y sesenta del siglo pasado se inició en Japón el uso de la inyección de silicona líquida como método para aumentar el volumen mamario ${ }^{6}$. Diferentes estudios posteriores han ido comprobando que no satisfacen todas las propiedades del tejido blando sintético ideal, y se ha informado de diversas complicaciones, más importantes en los casos de inyección de silicona líquida libre. La Food and Drug Administration estadounidense, que no había autorizado la inyección, terminó prohibiéndola en 1976. Pero la técnica se difundió en México y Asia ${ }^{6}$, y todavía hoy se siguen presentando de forma esporádica casos en los que se practica la técnica, generalmente de forma clandestina

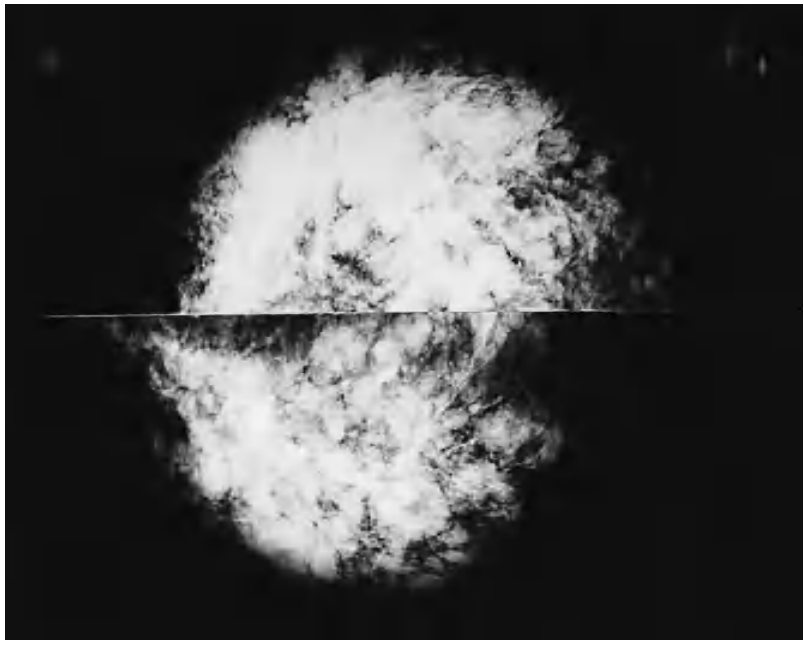

Fig. 1. Mamografía que muestra aumento de la densidad mamaria sin posibilidad de identificar la glándula.
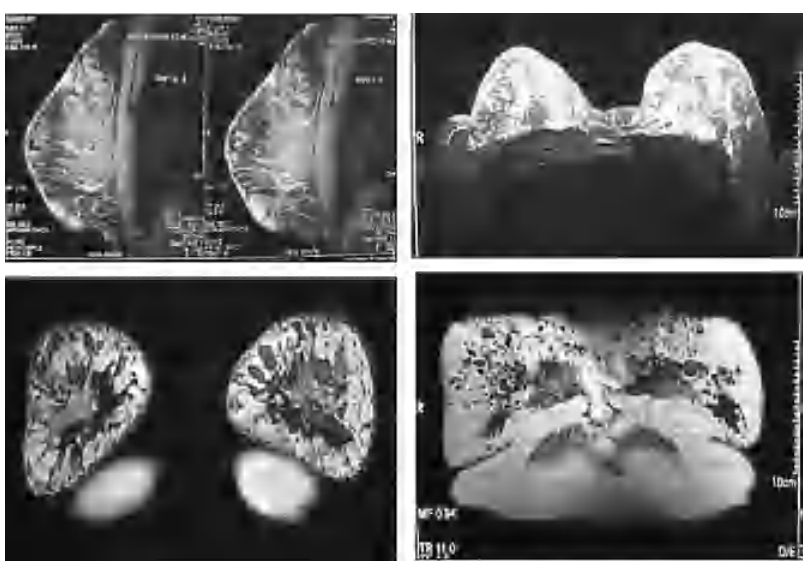

Fig. 2. Resonancia magnética en la que se observan múltiples imágenes ovoideas correspondientes a implantes de silicona.

en ambientes extramédicos, como los casos que presentamos.

El descrédito de la técnica nace, por una parte, de la dificultad para el seguimiento de las mamas así tratadas, $y$ de las manifestaciones locales y regionales de intolerancia que la silicona inyectada produce. Las dificultades que la silicona inyectada causa para la valoración de las técnicas de imagen habituales reducen de forma importante las posibilidades de un diagnóstico temprano del cáncer de mama.

Existen escasas referencias a la utilidad de la ecografía para la valoración de las mamas tras la inyección de silicona líquida. El fuerte refuerzo acústico provocado por la silicona dificulta el estudio mediante ultrasonidos. La alta resolución de la RM hace de esta técnica la más útil para el estudio de las mamas que se han sometido a la inyección de silicona líquida ${ }^{7}$. Como en nuestros casos, permite una cierta fiabilidad para la exclusión de patología tumoral, a la vez que aporta información sobre la difusión de la silicona más allá de la glándula 


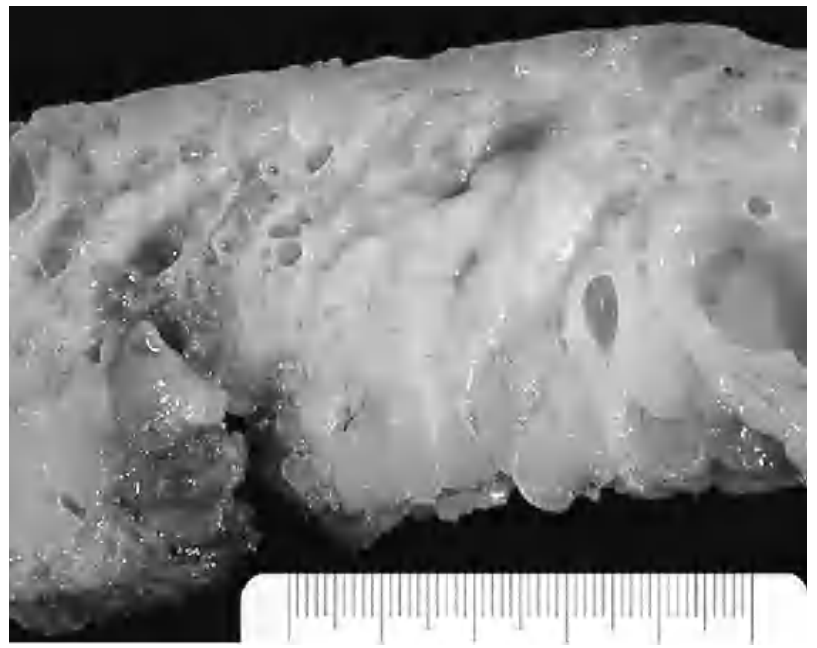

Fig. 3. Imagen macroscópica: múltiples cavidades quísticas con contenido oleoso entremezclado con el tejido adiposo mamario.

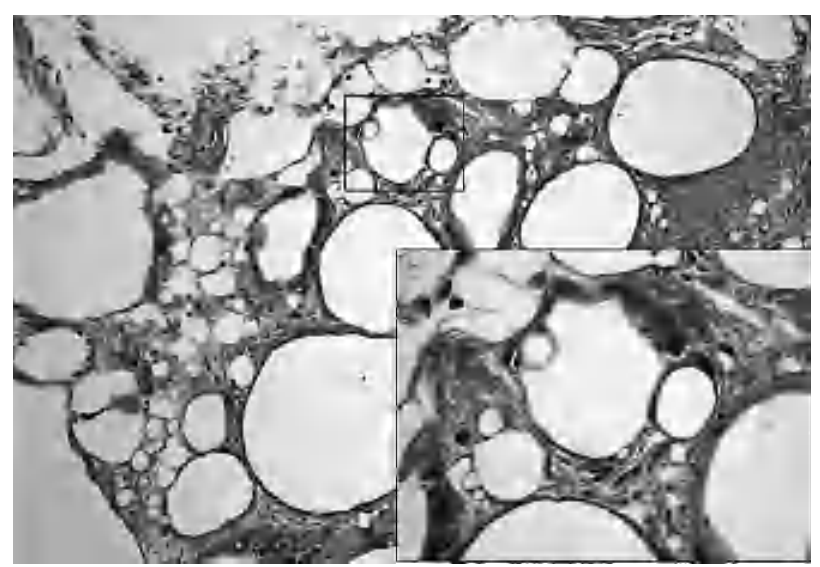

Fig. 4. Imagen microscópica: vacuolas con pequeña cantidad de tejido refringente en el interior de los macrófagos.

mamaria (ganglios regionales y tejidos vecinos a la mama).

No se ha podido observar un incremento en la incidencia de cáncer de mama en las mujeres a las que se ha inyectado silicona ${ }^{8}$, aunque se han descrito varios casos de carcinoma escamoso asentado en mamas a las que previamente se había inyectado silicona líquida². La ausencia de células de este tipo en la estructura mamaria justificaría la excepcionalidad de esta forma tumoral, y pueden aparecer en el contexto de una inflamación prolongada con cambios metaplásicos tras la inyección de silicona ${ }^{9}$. A esta dificultad para el diagnóstico de una posible neoplasia mamaria debe sumarse las frecuentes complicaciones, tempranas y tardías $^{8}$, descritas en los casos presentados, como el dolor mamario, las infecciones agudas, granulomas inflamatorios, mastitis crónica, retracciones cutáneas, fistulización de la silicona a piel, y su migración a ganglios regionales e incluso a zonas extramamarias ${ }^{10,11}$. La presencia de silicona en la zona gan- glionar axilar es un hallazgo accidental en pacientes sometidas a mastectomía después de mamoplastias de aumento, tanto por inyección de silicona libre como mediante prótesis de silicona ${ }^{12}$, y se manifiestan con dolor local o simplemente con aumento del tamaño ganglionar. En la última década se ha asistido a una importante controversia sobre la posible relación entre los implantes de silicona y la aparición de enfermedades del tejido conectivo, sin que se haya podido establecer una relación definitiva ${ }^{13}$

La alta incidencia de complicaciones locales generada por la inyección de silicona líquida hace recomendar la mastectomía, no sólo en el caso de masas mamarias sospechosas, sino también ante episodios repetidos de mastitis, dolor mamario o en el caso de historia familiar de cáncer de mama ${ }^{14}$. La técnica no es sencilla, dada la dificultad para extraer la totalidad de la silicona inyectada que se ha difundido por el tejido mamario y adyacente, especialmente cuando, como en nuestros casos, el volumen inyectado ha sido importante, generando una reacción inflamatoria con extensa necrosis en el tejido celular subcutáneo.

Por todo lo referido, y dada la posibilidad de recidiva de implantes de silicona, incluso en regiones diferentes de la zona de inyección que pueden precisar múltiples intervenciones, es recomendable el seguimiento de estas pacientes a largo plazo.

\section{Bibliografía}

1. Bridges AJ, Vasey FB. Silicone breast implants. History, safety and potential complications. Arch Inter Med. 1993;153:2638-44.

2. Smith LF, Smith TT, Yeary E, McGee JM, Malnar K. Squamous cellcarcinoma of the breast following silicone injection of the breasts. $J$ Okla State Med Assoc. 1999;92:126-30.

3. Ellenbogen R, Rubin L. Injection fluid silicone therapy: human morbidity and mortality. JAMA. 1975;234:308-9.

4. Hui-Ling $P$, Chin-Chi W, Wai-Man Ch, et al. Breast cancer detection using magnetic resonance imaging in breast injection with liquid silicona. Plast Reconst Surg. 1999;104:2116-20.

5. Steinbach BG, Hardt NS, Abbitt PL. Mammography: breast implants, types, complications and adjacent breast pathology. Curr Probl Diagn Radiol. 1993;22:39.

6. Hardt NS. latrogenic lesions of the breast. En: Silverberg SG, editor. Atlas of breast pathology. Philadelphia: Saunders Comp; 2002.

7. Peng HL, Wu CC, Choi WM, Hui MS, Lu TN, Chen LK. Breast cancer detection using magnetic resonance imaging in breasts injected with liquid silicone. Plastic Reconstr Surg. 1999;104:2116-20.

8. Liebman AJ, Sybus R. Mammographic and sonographic fundings after silicone inyection. Ann Plast Surg. 1994;33:412-4.

9. Talmor M, Rothaus KO, Shannahan E, Cortese AF, Hoffman LA. Squamous cell carcinoma of the breast after augmentantation with liquid silicone injection. Ann Plast Surg. 1995;34:619-23.

10. Komenaka IK, Ditkoff BA, Schnabel F, Marboe ChC, Mercado C. Free silicone injection causing polyarthropathy and septic shock. Breast J. 2004;10:160-1.

11. Wang J, Shih TT, Chang KJ, Li YW. Silicone migration from silicone injection breast: magnetic resonence images Ann Plast Surg. 2002; 48:617-21.

12. Truong LD, Cartwright J, Goodman MD, Woznicki D. Silicone lymphadenopathy with augmentation mammoplasty. Morphologic features of nine cases. Am J Surg Pathol. 1988;12:484-1.

13. Janowsky EC, Kupper LL, Hulka BS. Meta-analyses of the relation between silicone breast implants and the risk of connective-tissue diseases. N Engl J Med. 2000;342:781-90.

14. Ko C, Ahn CY, Markowitz BL. Injected liquid silicone, chronic mastitis and undetected breast cancer. Ann Plast Surg. 1995;34:176-9. 